\title{
ORGANIZATIONAL ATTRACTION
}

\author{
Eva Derous \& Lien Wille
}

Ghent University

\section{Reference:}

Derous, E., \& Wille, L. (2017). Organizational attraction. In S. Rogelberg, K. Shockley, \& S. Toninandel (Eds.), The SAGE encyclopedia of industrial and organizational psychology (2nd Edition, Vol. 3) (pp. 1060-1064). New York: Sage.

DOI: $10.4135 / 9781483386874$ - ISBN: 9781483386898 


\section{ORGANIZATIONAL ATTRACTION}

\section{Concept and Measurement}

Since the beginning of the nineteens, organizational attraction has emerged as a topic of great interest to both practitioners and academics. Different definitions of organizational attraction have been formulated, all referring to individuals' affective and attitudinal thoughts about particular organizations as potential places for employment. Implicitly -if not explicitlydefinitions on organizational attraction stress job seekers' perceptions of organizations as desirable, positive places to work for. Organizational attraction has been measured in both direct and indirect ways. Examples of direct measures of organizational attraction include behavioralbased measures among which one's recommendation of organizations to others, one's ultimate choice of an organization, and even actual application behavior. Typically, however, organizational attraction is measured indirectly through the use of a single item or scale with three to five items. Although the difference is subtle, some items seem to operationalize organizational attraction in more general terms than others. Some items, for instance, reflect job seekers and applicants' general appreciation of organizations ("[Company X] is one of the best employers to work for"). Other items capture specific intentions or the extent to which job seekers and applicants feel personally attracted to work for a particular organization ("How much do you like to work for [Company X]").

Recent decades have witnessed an exponential growth of studies on organizational attraction. One reason is that organizational attraction seems to be a critical component to the success of organizations. Its importance relates to the effect of organizational attraction on factors that affect the overall productivity of organizations, among which the attraction and selection of highly competent and productive employees. Hence, organizational attraction is a vital theme in many companies' human resources management, and in particular recruitment and selection 
practices. Not surprisingly, much attention has gone to job seekers' affective and attitudinal thoughts about organizations they want to initiate a relation with, in order to optimize organizations' reputation and recruitment practice.

Criticism, however, has been expressed regarding the lack of construct clarity. As illustrated, organizational attraction has been defined in a rather broad way. Several concepts are related to the notion of organizational attraction and even used intertwined, among which applicant attraction, job attraction, person-organization fit (P-O fit) person-job fit (P-J fit), organizational reputation, organizational image, and job pursuit intentions. Below, we describe how these notions relate to organizational attraction.

Applicant attraction involves applicants' overall evaluation of the attractiveness of work opportunities including many job and organizational characteristics. Hence, in recent metaanalyses, organizational attraction has been considered as an index of applicants' attraction but has also been used as a substitute for applicant attraction because of their relatively high intercorrelations (in the range of .60-.70). Job attraction, on the other hand, refers to one's overall evaluation of the attractiveness of a particular job and its characteristics ("How attractive is this job to you?"). As jobs are typically performed within an organizational setting, recent meta-analyses have considered job attraction as an index of organizational attraction.

Person-organization fit ( $P$-O fit) covers an individual's perceived or actual congruence with an organization. For instance, individual's personal characteristics might complement or supplement an organization's system, structures, values, and culture (“The values of this organization are similar to my own values”). Similarly, person-job fit ( $P$-J fit) reflects the perceived or actual congruence of a person's characteristics with a particular job ("To what extent do your knowledge, skills, and abilities "match" or fit the requirements of the job?”). Both P-J fit and P-O fit notions are determinants of organizational attraction (see further) with empirical 
studies showing perceived/subjective and actual/objective P-O fit to be a somewhat stronger correlate of organizational attraction (in the range of .40-.60) than perceived and actual P-J fit measures (in the range of .40-.50).

Organizational reputation comprises a collective of individual impressions regarding an organization's relative standing externally (with clients and other external stakeholders) and internally (with employees and internal stakeholders) in the organization's business environment. Knowledge of an organizational reputation is expected to affect organizational attraction, and hence considered as a determinant of organizational attraction. Correlations between organizational attraction and reputation or prestige measures ("This is a reputable company to work for") are typically moderate to high (around .40).

Relatedly, one of the most important correlates of organizational attraction is the organization's image (in the range of .40-.50). In line with the instrumental-symbolic framework -as adopted from marketing research- organizational image refers to both instrumental and symbolic attributes of the organization as determinant of the organization's attractiveness. Instrumental attributes entail individuals' perceptions of tangible, concrete job and organizational characteristics, including for instance job security, advancement opportunities, pay, task demands, and organizational location ("People who work in [Company X] have a solid job"). Symbolic attributes refer to more abstract, intangible characteristics that people infer from how they experience organizations. Symbolic attributes are typically measured with a list of adjectives and refer to the organization's personality: "If I were to consider [Company X] as a person, I would describe it as [adjective]". Examples of trait inferences are innovative, competent, tough.

Finally, organizational attraction has been used as a surrogate measure of job pursuit intentions which entails whether one intends to pursue application in an organization ("If this company invited me for a second job interview, I would go"). Empirical studies have evidenced 
high intercorrelations but still conceptual differences between organizational attractiveness and job pursuit intentions (in the range of .60-.80). From a conceptual point of view and in line with the theory of planned behavior, organizational pursuit intentions are considered as an outcome variable of organizational attraction, which in turn has been conceptualized as its' attitudinal antecedent.

\section{Theoretical Perspectives}

Besides the need for construct clarity, the organization attraction literature has also been criticized for its a-theoretical nature. Since the mid-2000s, however, systematic efforts have been made to investigate the theoretical underpinnings of individuals' attraction to organizations. Three clusters of well-known psychological theories (i.e., meta-theoretical explanations) have been put forward to explain mechanisms of individuals' attraction to organizations.

A first group of theories discusses how individuals process information about their environment, which in turn affects their attraction to organizations (environment processing metatheory). An example is signaling theory, which states that applicants process information about the organization as signals of organizational characteristics that affect attraction. For instance, recruiters' warmth may be perceived as a signal of a considerate and warm workplace, hence increasing applicants' attraction to that organization. A second group of theories proposes that individuals evaluate fit between their own personal characteristics (like personal needs, values) and those from the environment (interactionist processing metatheory). An example is the attraction-selection-attrition theory, which states that individuals will be attracted to organizations that have certain characteristics they feel a 'match' with but will leave organizations if they feel 'no match' anymore. Finally, a third group of theories discusses how information about the self (like self-esteem) moderates perceived P-O fit and individuals' perceived organizational attractiveness (self-processing metatheory). According to the 
consistency theory, for instance, individuals with a high self-esteem would prefer organizations that fit their needs and correspond to their self-image.

Some recently formulated models build upon assumptions expressed by these three metatheories, hence showing the complex and multifaceted nature of organizational attraction. For instance, the theory of symbolic attraction suggests that symbolic inferences mediate companies' market signals (like company rankings) and applicants' attraction to organizations (environment processing metatheory) but that any effect of symbolic inferences on organizational attraction also depends on applicants' social identity concerns (interactionist and self-processing metatheories).

Researchers have started validating these mechanisms of organizational attraction. Remarkably, most theoretical models and studies are rather 'static' in nature, considering organizational attraction at a single point in time. However, applicants' evaluation of organizational attributes might change throughout the recruitment process (e.g., as a function of recruitment stage) and throughout individual careers (e.g., as a function of professional experiences and personal developments). Future research, therefore, could approach organizational attraction in more dynamic ways by investigating mechanisms of change as well as conditions under which perceptions of organizational attraction would be revised or modified.

\section{Antecedents and Consequences}

The literature on organizational attraction has grown fast and empirical research has investigated job seekers and applicants' reactions towards a plethora of job and organizational characteristics. While it is beyond the scope of this entry to review all of the literature, presented below are some key conclusions regarding antecedents and consequences of organizational attraction in the workplace. Antecedents of organizational attraction have received a lot of research attention in the past decades and are rooted in both the organization and the individual, as discussed next. 
Organizational determinants. Many organizational characteristics have been documented as desirable to applicants, among which the organization's structure (like company's degree of decentralization) and policies (from workplace romance policies to the implementation of diversity programs). As mentioned, one of the strongest determinants seems to be the organization's image, including the organization's instrumental/symbolic attributes. Other types of image concepts relate to product image and corporate social responsibility (CSR or the organization's community involvement and pro-environmental practices). In general, applicants feel more attracted to organizations that help employees/society to realize their (personal) goals, show high concern for others, and display organizational fairness.

In addition, a lot of research attention has gone to recruitment-related sources and selection practices. Recruiters' warmth and professionalism, for instance, seem to strongly affect applicants' organizational attraction. On the contrary, unfairness (like discriminatory interview questions) and privacy-invasiveness (like screening of applicants' social network sites) negatively affect organizational attraction. Types of recruitment media have also been considered. In general, applicants prefer organizations that make use of modern, technologydriven tools. Hence, effects of technological developments like e-recruitment (e.g., websites; recruitment games) are a topic of great interest. Interestingly, less technology-driven or sophisticated tools like word-of-mouth communication, seem also to work, particularly if the source of employment information is perceived as credible. Finally, job attributes and working conditions (like individual-based pay) and career paths that organizations offer (like flexible career paths) have been demonstrated to positively affect organizational attraction.

Taken together, organizational policies and HR practices that stress investment in people and society (like training and development; CSR) are most favorably regarded. Symbolic attributes (like trait inferences) further account for incremental variance over concrete job and 
organizational attributes in predicting organizational attraction. In terms of the relative strength, a recent meta-analysis further showed organizational characteristics (like image and reputation) to be a stronger determinant than job characteristics (like benefits), followed by a group of determinants that appeared to be equally strong determinants among which recruiter characteristics and characteristics of the recruitment process (all medium-sized effects). Importantly, some determinants, like job characteristics, seemed to have stronger effects in field than in lab settings whereas the reverse was found for characteristics of the recruiter and the recruitment process.

Individual, person-based determinants. Whether organizational characteristics are considered as desirable seems, however, contingent on the degree of fit with applicant characteristics, which corroborates with interactionist perspectives on organizational attraction. Indeed, recent meta-analyses support this assumption, given that perceived fit (P-O/P-J) seemed the strongest correlate of applicant attraction (with large effects) compared to organizational characteristics (medium-sized effects). Among individual characteristics that have been investigated are individuals' values and needs (like need for materialism or a warm environment), demographic characteristics (like ethnicity), personality characteristics (like conscientiousness, openness to experience), and several other individual difference factors (like one's social identity concerns and self-esteem). For instance, the degree that individual-based pay affects organizational attraction seems to depend on individuals' standing on materialism. Organizations offering flextime are more appealing to those with high role conflict and effects of CSR on organizational attraction seem to be moderated by applicants' level of Machiavellism. Interestingly, these examples do not measure actual P-O fit but validate correlations between perceived P-O fit and organizational attraction measures. 
Consequences. Finally, organizational attraction seems to have important consequences

for both organizations and individuals. Specifically, organizational attraction has been related positively to several behavioral and attitudinal outcome variables, among which applicants' job pursuit intentions, job acceptance intentions ("If you were offered the job, would you accept it?"), job choice (operationalized as actual job acceptance, i.e., whether one accepts a real job offered by a company), and individuals' affective commitment to organizations (“I feel emotionally attached to [company X]”). Although empirical evidence is limited, organizational attraction is even suggested to add competitive value by increasing employee and customer satisfaction. Companies, therefore, could engage in a thorough auditing and marketing of their organizational image and reputation. Building further on the fit idea, then, other practical implications comprise the use of targeted recruitment practices to attract particular groups of applicants.

\section{Conclusion}

Lately, a lot of research attention has gone to organization attraction given its key importance in organizations. In this entry, we provided a description of organizational attraction and related notions, the way attraction has been measured, theoretical viewpoints on underlying mechanisms, and its correlates as being reported in the research literature over the past two decades. Overall, study findings highlight the complex, subjective, and multifaceted nature of organizational attraction. Future research could investigate ways to improve organization attraction, and might benefit from employing a more dynamic perspective.

\section{Cross-references (suggestions)}

job choice; organizational image; person-organization fit; person-job fit; recruitment; recruitment sources 


\section{Recommended readings}

Chapman, D. S., Uggerslev, K. L., Carroll, S. A., Piasentin, K. A., \& Jones, D. A. (2005). Applicant attraction to organizations and job choice: A meta-analytic review of the correlates of recruiting outcomes. Journal of Applied Psychology, 90, 928-944. doi: $10.1037 / 0021-9010.90 .5 .928$

Ehrhart, K. H., \& Ziegert, J. C. (2005). Why are individuals attracted to organizations? Journal of Management, 31, 901-919. doi:10.1177/0149206305279759

Highhouse, S., Lievens, F., \& Sinar, E. F. (2003). Measuring attraction to organizations. Educational \& Psychological Measurement, 63, 986-1001. doi:10.1177/0013164403258403

Lievens, F., \& Highhouse, S. (2003). The relation of instrumental and symbolic attributes to a company's attractiveness as an employer. Personnel Psychology, 56, 75-102. doi :10.1111/j.1744-6570.2003.tb00144.x

Schreurs, B., Derous, E., Van Hooft, E. A. J., Proost, K., \& De Witte, K. (2009). Predicting applicants' job pursuit behavior from their selection expectations: The mediating role of the theory of planned behavior. Journal of Organizational Behavior, 30, 761-783. doi:10.1002/job.570

Uggerslev, K. L., Fassina, N. E., \& Kraichy, D. (2012). Recruiting through the stages: A metaanalytic test of predictors of applicant attraction at different stages of the recruiting process. Personnel Psychology, 65, 579-660. doi:10.1111/j.1744-6570.2012.01254.x

Walker, H. J., Feild, H. S., Giles, W. F., Armenakis, A. A., \& Bernerth, J. B. (2009). Displaying employee testimonials on recruitment web sites: Effects of communication media, employee race, and job seeker race on organizational attraction and information credibility. Journal of Applied Psychology, 94, 1354-1364. doi:10.1037/a0014964 
Yu, K. Y. T. (2014). Person-organization fit effects on organizational attraction: A test of an expectations-based model. Organizational Behavior and Human Decision Processes, 124, 75-94. doi:10.1016/j.obhdp.2013.12.005 